\title{
Chinese Medicine in the Battle Against Obesity and Metabolic Diseases
}

\author{
Lingyan Xu, Wenjun Zhao, Dongmei Wang and Xinran Ma*
}

Shanghai Key Laboratory of Regulatory Biology, Institute of Biomedical Sciences, School of Life Sciences, East China Normal University, Shanghai, China

Obesity is a multi-factor chronic disease caused by the mixed influence of genetics, environments and an imbalance of energy intake and expenditure. Due to lifestyle changes, modern society sees a rapid increase in obesity occurrence along with an aggravated risk of metabolic syndromes in the general population, including diabetes, hepatic steatosis, cardiovascular diseases and certain types of cancer. Although obesity has become a serious worldwide public health hazard, effective and safe drugs treating obesity are still missing. Traditional Chinese medicine (TCM) has been implicated in practical use in China for thousands of years and has accumulated substantial front line experience in treating various diseases. Compared to western medicine that features defined composition and clear molecular mechanisms, TCM is consisted with complex ingredients from plants and animals and prescribed based on overall symptoms and collective experience. Because of their fundamental differences, TCM and western medicine were once considered irreconcilable. However, nowadays, sophisticated isolation technologies and deepened molecular understanding of the active ingredients of TCM are gradually bridging the gap between the two, enabling the identification of active TCM components for drug development under the western-style paradigms. Thus, studies on TCM open a new therapeutic avenue and show great potential in the combat against obesity, though challenges exist. In this review, we highlight six key candidate substances derived from TCM, including artemisinin, curcumin, celastrol, capsaicin, berberine and ginsenosides, to review their recent discoveries in the metabolic field, with special focus on their therapeutic efficacy and molecular mechanisms in treating obesity and metabolic diseases. In addition, we discuss the translational challenges and perspectives in implementing modern Chinese medicine into the western pharmaceutical industry.

This article was submitted to Lipid and Fatty Acid Research, a section of the journal

Frontiers in Physiology

Received: 09 March 2018 Accepted: 14 June 2018 Published: 06 July 2018

Citation: Xu L, Zhao W, Wang $D$ and Ma X (2018) Chinese Medicine in the Battle Against Obesity and Metabolic Diseases. Front. Physiol. 9:850. doi: 10.3389/fphys.2018.00850

Abbreviations: AMPK, Protein kinase AMP-activated catalytic subunit alpha 1; ATF2, Activating transcription factor 2; C/EBP, CCAAT/enhancer binding protein; HO-1, Heme oxygenase-1; HSF1, Heat shock factor 1; LDLR, Low density lipoprotein receptor; MAPK, Mitogen activated kinase-like protein; NRF2, Muclear factor erythroid 2; PGC1 $\alpha$, Peroxisome proliferative activated receptor gamma coactivator 1 alpha; PPAR $\gamma$, Peroxisome proliferator activated receptor gamma; PRDM16, PR/SET domain 16; Sirt1, Sirtuin 1; SREBP1, Sterol regulatory element binding protein 1; STAT3, Signal transducer and activator of transcription 3; TPRM8, Transient receptor potential cation channel subfamily M member 8; TRPA1, Transient receptor potential cation channel subfamily A member 1; TRPV1, Transient receptor potential cation channel subfamily V member 1; TRPV4, Transient receptor potential cation channel subfamily V member 4; UCP1, Uncoupling protein 1. 


\section{INTRODUCTION}

For thousands of years, before the introduction of western medicine, Chinese people relied on traditional Chinese medicine (TCM) to treat diseases and relieve discomfort. TCM is complex formula constituted of mixed medicinal extracts from Chinese herbs and animals, and is prescribed based on the philosophical theory of Chinese medicine, for instance, the theories of "Yin Yang" and "Five Elements." Though mysterious and empirical, TCM has gained front line experience in combating diseases for millennia in Chinese history with effectiveness and low side effects. However, in the modern society, with the prevalence of western medicine, which is developed under strict experimental proofs and feature clearcut molecular compositions and mechanisms of action, TCM meets serious criticisms and challenges due to undefined compositions and unclear therapeutic mechanisms (Qiu, 2007). Fortunately, nowadays, sophisticated isolation techniques have enabled scientists to identify active ingredients from TCM to elucidate the molecular mechanisms of their therapeutic effects, thus promote the re-discovery of ancient TCM compounds to be potentially developed as drugs under western medical standard. A few promising TCM compounds with successful implications include, but not limit to, artemisinin in treating malaria (Liu et al., 2006) and arsenic trioxide in acute promyelocytic leukemia (Lo-Coco et al., 2013), with more waiting to be added to the list.

Looking back into the history of human diseases, before the enlightenment of modern medicine and vaccinology, infectious disease outbreaks decimated large populations and were once the most threatening events to mankind. In today's society, however, with large scale vaccination and a highly organized disease control system, the biggest challenge to human health has shifted to noninfectious chronic disease (NCD) like obesity, metabolic diseases, cardiovascular diseases, neurodegenerative diseases, inflammatory diseases and various types of cancer. Among them, obesity holds special importance as it is a major risk factor for many other metabolic diseases, i.e., type 2 diabetes mellitus, nonalcoholic fatty liver disease, hyperlipidemia, etc., thus renders it an effective target for disease intervention (Haslam and James, 2005). Briefly, obesity is an excessive deposit of white fat in the body. The abnormal accumulation of fat poses adverse impacts on the metabolic fitness of the body and is positively associated with aberrant metabolic complications including hyperglycemia, insulin resistance, dyslipidemia, hypertension (Bays and Dujovne, 2006) and chronic low-grade inflammation (Saltiel and Olefsky, 2017). Fat tissues are metabolically active organs and are classified as white, brown and beige fat based on their morphology, physiology and functions (Boss and Farmer, 2012; Xu et al., 2018). Together they maintain the energy balance of the body with white fat storing energy in forms of triglycerides, while brown and beige fat dissipating energy for thermogenesis via UCP1 activation. The disturbance of energy balance among adipose tissues may lead to increased adiposity and/or obesity.

Considering different therapeutic leads, compounds derived from TCM possess two major advantages over synthetic drugs in treating obesity. First, obesity is a complex disease that shows no clear pathogens and its pathogenesis involves multiple genetic factors and signaling networks. In patients with obesity and metabolic diseases, multiple organs, i.e., fat tissues, liver, muscle, and even brain suffer pathological changes. In this sense, the concept of TCM emphasizes the holistic treatment of a disease. Studies on TCM have demonstrated that their active ingredients target multiple organs and tissues in the body to exert systemic effects, which match the characteristics of obesity. Second, obesity and metabolic diseases are not as immediately crippling or death threatening as infectious diseases. Patients require long-term care and persistent treatment to prevent advance disease progression, which greatly burdens their family and the society as a whole. Thus, it is important to take long-term effectiveness and safety into account when developing drugs for obesity. In this regard, most TCM consists of herbal-based medicinal extracts that have been prescribed and widely used in Chinese people's daily life for purposes ranging from health-promoting to disease treatment for thousands of years, which provide solid though empirical evidence for its safety. These characteristics make TCM a promising source for new drug development for obesity and metabolic diseases. However, putting their appealing efficacies aside, the caveat is that much of TCM has unclear compositions and vague mechanisms, which not only hinders their wide application into modern clinic, but can sometimes be detrimental. One extreme case is the recently reported implication of aristolochic acids and similar compounds from Aristolochia and related plant in liver cancers in Asia population for their mutagenesis attribution ( $\mathrm{Ng}$ et al., 2017). Thus, it is vital to place TCM in the pipeline of standard western drug development: identification, isolation and/or synthesis of the active component, clarification of the molecular target and mechanism, and eventually developed as a pharmaceutical (Corson and Crews, 2007). During this process, various factors like therapeutic mechanism, clinical data, development cost, etc. all play important roles. In recent years, great interests have been focused on TCM, producing a substantial amount of research data. In this review, we highlight recent studies of six promising TCM compounds in the metabolic aspect based upon their clinical, mechanistic properties or current application, including artemisinin, curcumin, celastrol, capsaicin, berberine and ginsenosides.

\section{ACKNOWLEDGED VS. CONTROVERSIAL: ARTEMISININ AND CURCUMIN}

Although the interests in TCM's potential in treating obesity and metabolic diseases only start to rise in recent years, many TCM ingredients have already been isolated and tested over the years. Substantial data demonstrates their effectiveness and low adverse effects in various diseases, promoting a few into preclinical or clinical trials, though not all trials gave consistent results. Among them, artemisinin and curcumin represent two different extremes, one's efficacy is well acknowledged while the other is shrouded in controversy. 


\section{Artemisinin}

Artemisinin is derived from the sweet wormwood (Artemisia annua L.) that is regularly used as classic TCM. Artemisinin and its semi-synthetic derivatives-based therapy are currently the standard and the most effective treatment for uncomplicated Plasmodium falciparum malaria. In 2015, Dr. Youyou Tu was awarded the Nobel Prize for medicine for discovering and isolating artemisinin. This makes artemisinin the most acknowledged and successful TCM compound.

Aside from malaria, in recent years, with the spike in overweight and obesity rate, more and more attentions are focusing on the roles of artemisinin and its derivatives in treating obesity and metabolic diseases. For example, artemisinic acid and artesunate are found to inhibit the development and differentiation of adipocytes by suppressing master regulators C/EBPs and PPAR $\gamma$ in adipogenesis (Lee et al., 2012; Jang, 2016). In addition, since the groundbreaking discovery of brown and beige fat in human adults, activation of brown fat and browning of white fat, two major sources of adaptive thermogenesis and important outputs for energy expenditure, have emerged as potential therapeutic means to treat obesity and metabolic diseases (Cypess and Kahn, 2010; Boss and Farmer, 2012). By high-throughput screening over 3000 compounds in differentiated 3T3-L1 and $\mathrm{C} 3 \mathrm{H} 10 \mathrm{~T} 1 / 2$ adipocytes to find small-molecule compounds capable of activating thermogenesis, $\mathrm{Lu}$ et al have identified artemether, an artemisinin derivative, as an activator of browning and thermogenesis in vitro. Further examination reveals artemisinin and other artemisinin derivatives, dihydroartemisinin, artesunate and arteether, could also promote browning. Importantly, local delivery of artemether into subcutaneous fat or systemic delivery via tail vein in mice effectively reduces high fat diet induced body weight gain, enhances cold tolerance and improves insulin sensitivity. Mechanistic study indicates that p38 MAPK/ATF2 axis and Akt/mTOR pathway are partially responsible for the browning effects of artemether ( $\mathrm{Lu}$ et al., 2016). In another study, it is reported that leaf extracts of Artemisia annua, the source of artemisinin, attenuates hepatic steatosis and inflammation in diet-induced obese (DIO) mice (Kim et al., 2016). These studies shed first light on the possibility of exploiting artemisinin and its derivatives as an anti-obesity and anti-fatty liver drug in vivo, though detailed mechanistic study and clinical data are warranted. As a paradigm of the translational application of TCM, continued characterization of the metabolic properties of artemisinin and its derivatives would bring hope to patients with obesity and metabolic diseases.

\section{Curcumin}

In sharp comparison with artemisinin, whose effectiveness is well acknowledged in academia, curcumin may be the most controversial TCM compound in the eyes of western chemists and biologists. Curcumin is the principal curcuminoid in the turmeric of the ginger family. There are thousands of reports and over 120 clinical trials studying the effectiveness of curcumin in various diseases, including erectile dysfunction, hirsutism, baldness, cancer, and Alzheimer's disease, yet the results fail to reconcile with one another. The contradictions are probably due to the poor solubility of curcumin in aqueous solution, thus limiting its bioactivity and stability in many studies (Nelson et al., 2017). When interpreting results from various studies, one has to keep in mind that, on one hand, substantial evidences exist in a large body of literatures about curcumin's biological activities under different circumstances and its effectiveness both in vitro and in vivo (Heger, 2017). But on the other hand, it is of major concern that curcumin fluoresces naturally, which may interfere with drug screening that relies highly on fluorescence signals, producing screen artifacts and generating false positive results (Baker, 2017). Thus, any attempt at the pharmacological use of curcumin should take these factors into account.

In the metabolic aspects, in adipocytes, consistent reports indicate that curcumin suppresses adipocyte differentiation by affecting classic regulators of adipogenesis (Ejaz et al., 2009; Kim et al., 2011; Sakuma et al., 2017). Curcumin also ameliorates hypoxia-induced insulin resistance and inflammation in 3T3L1 adipocytes (Priyanka et al., 2017). In the browning process, it has been shown that curcumin treatment induces browning in primary white adipocytes and adipose tissues and augments thermogenic and mitochondrial gene programs in a classic norepinephrine dependent manner (Wang et al., 2015; Lone et al., 2016). In rodents, curcumin treatment enhances energy expenditure in DIO murine models and protects against weight gain and inflammation of adipose tissues (Weisberg et al., 2008; Shao et al., 2012). In a few clinic trials, curcumin has been shown to improve insulin resistance and hyperlipidemia in patients with metabolic syndromes (Mohammadi et al., 2013; Ganjali et al., 2014), while others produce negative results (Nelson et al., 2017).

Importantly, the resolution to the predicament of insolubility and instability of curcumin, which hinders its clinical application, may lie in the researches of curcumin analogs. For example, curcumin-3, 4-dichloro phenyl pyrazole (CDPP) shows significantly improved bioactivity while retains curcumin's capability in suppressing adipocyte differentiation and preventing hyperlipidemia in DIO rodents (Gupta et al., 2017). C66, a novel curcumin derivative, inhibits JNK phosphorylation, reduces high glucose-induced inflammation in cardiomyocytes and prevents the development of diabetic cardiomyopathy in mice (Pan et al., 2014). Alternatively, nano-formulated curcumin generated using nanoparticles has shown improved bioactivity and efficacy compared to native curcumin in various in vitro and in vivo disease models, indicating its potential implication in metabolic studies (Rahimi et al., 2016). However, despite the metabolic benefits in curcumin-treated adipocytes and mice models, these studies lack detailed mechanisms and target molecules. Further mechanistic investigations are needed to disperse the controversy around curcumin before it can be further implicated in the treatment of obesity and metabolic diseases.

\section{MULTI-TARGETS VS. SINGLE TARGET: CELASTROL AND CAPSAICIN}

Mechanistic studies are vital in drug development to prevent potential side effects. Compounds with single targets are favored 
by scientists for their balanced efficacy and safeness, although compounds with multiple targets in multiple organs could be of potential interest in treating obesity and metabolic diseases since the patients usually feature pathological changes in not one but multiple organs, i.e., adipose tissues, liver and muscle. As an example, celastrol is a TCM compound targeting multiple metabolic organs with detailed mechanisms of action deciphered (Liu et al., 2015; Ma et al., 2015; Hu et al., 2017). In comparison, capsaicin is well known for functioning through its receptor TRPV1 (Caterina et al., 1997), the mechanism of which has been extensively studied.

\section{Celastrol}

Celastrol (tripterine) is a chemical compound isolated from the root extracts of Tripterygium wilfordii (Thunder god vine, TGV). As a TCM, TGV has wide and longtime application in treating inflammatory diseases in patients, i.e., rheumatoid arthritis. As one of the major active ingredients of TGV, celastrol has multiple functions in anti-oxidation, antiinflammation, anti-neurodegeneration, and anti-cancer, though its anti-inflammation effects are most extensively studied (Cascão et al., 2017). Recently, a series of studies have brought celastrol onto the metabolic stage and highlight it as a versatile regulator of obesity and metabolic diseases with both central and peripheral targets. Centrally, Liu et al. (2015) has reported that celastrol functions as a leptin sensitizer to reduce food intake in DIO mice and possibly treats obesity by both activating leptin receptor-STAT3 pathway and inhibiting NF- $\kappa B$ in the hypothalamus. Peripherally, Zhang's group has demonstrated that under inflammatory conditions, celastrol binds to Nur77, an orphan nuclear receptor and an inducer of mitochondria apoptosis, and promotes its translocation into mitochondrial for inflamed mitochondrial autophagy and clearance, thus reduces inflammation and improves hepatic steatosis in mice (Hu et al., 2017). Celastrol could also induce Sirt 1 transcription and alleviate hepatic steatosis by decreasing SREBP1 and increasing AMPK $\alpha$ expression (Zhang et al., 2016). Another report from Luo et al. (2017) reveals that in liver and adipose tissues of DIO mice, celastrol suppresses inflammation by reducing macrophage M1 polarization via its regulation on the Nrf2/HO-1, MAPK, and NF-кB pathways.

Apart from its impact on obesity and metabolic diseases through inflammatory inhibition in multiple organs, celastrol also exerts its beneficial effects by directly promoting energy expenditure. HSF1 is a classic transcription factor induced by multiple stimuli, including heat shock, oxidative and mechanical stresses (Anckar and Sistonen, 2011). It orchestrates the function of heat shock proteins in protein refolding and damage repair, thus is vital for protein homeostasis and cell survival (Gomez-Pastor et al., 2018). Interestingly, HSF1 could promote mitochondrial biogenesis and adaptive thermogenesis via its interaction with and transactivation of PGCl $\alpha$, the master regulator of energy metabolism that was previously thought to be mainly induced by cold exposure (Ma et al., 2015). Celastrol activates HSF1 as shown by HSF1 phosphorylation and activation of its downstream target genes (Westerheide et al., 2004). Based on these preliminary data and the fact that celastrol is derived from TCM with wide application in humans, Ma et al. (2015) test its metabolic effects and reveal that celastrol prevents weight gain, improves hepatic steatosis and ameliorates insulin resistance in mice fed a high fat diet. Intriguingly, in this study, celastrol blocks obesity progression in mice without reducing food intake or affecting hypothalamus metabolic genes expression, possibly because these mice didn't develop severe leptin resistance as the DIO model used in Liu's paper. Instead, celastrol activates the HSF1-PGC1 $\alpha$ axis in peripheral organs to induce browning of white fat and promote mitochondrial function in muscle. Celastrol treatment loses these beneficial effects in HSF1 and PGC1 $\alpha$ deficient cells and mice, suggesting that HSF1-PGC1 $\alpha$ axis is at least partially responsible for the metabolic function of celastrol in the peripheral organs (Ma et al., 2015).

In patients with obesity and metabolic diseases, multiple organs, i.e., adipose tissues, liver, muscle and brain experience pathological changes. It has been shown that celastrol targets multiple organs and improves their metabolic performances simultaneously, thus rendering it a TCM compound with comprehensive effects in treating obesity and metabolic diseases (Ma et al., 2015). Future work could be focused on its structural modification to obtain celastrol derivatives that preferentially target specific organs. Besides, it is of note that triptolide, another major active component of Tripterygium wilfordii, shows similar anti-inflammatory and anti-cancer effects to celastrol ( $\mathrm{Li}$ et al., 2014). Combined treatment of triptolide and celastrol shows synergistic effects in suppressing tumor cell growth in vitro (Jiang et al., 2015). It would be worthwhile to test their synergistic effects in the treatment of obesity and metabolic diseases. Finally, although celastrol treatment of various concentrations and lengths of time shows no overt side effects or toxicity in rodents, low sperm density and some toxicity are reported in male patients treated with TGV for rheumatoid arthritis (Lopez et al., 2005). Future studies are warranted to elucidate the efficacy and a safe concentration of action for celastrol and other TGV constituents in clinic.

\section{Capsaicin}

One hundred years ago, Capsaicin was identified and purified as one of the major active biological components of peppers (Capsicum). Peppers taste spicy by inducing the "hot" sensation to different extents and are widely used in daily life as a seasoning. They also have longtime use in TCM to relieve physical pain and treat gastrosis, detrusor hyperreflexia and rheumatic arthritis. Among active TCM compounds translated for pharmaceutical use, capsaicin is one of the best examples featuring extensively studied molecular targets and mechanisms. Unlike celastrol, which exerts its function through multiple targets in diverse organs, capsaicin functions through a single and clear target, its receptor TRPV1 (Caterina et al., 1997). TRPV1, a Ca ${ }^{2+}$ ion channel highly enriched in a specific subset of peptidergic sensory neuron in human and rodents, was identified and cloned in 1997 (Gunthorpe and Szallasi, 2008). It is a prime target for pain relief upon activation by capsaicin, as well as thermal heat, acidic conditions and allyl isothiocyanate (Gavva, 2008). TRPV1 antagonists reduce pain by blocking receptor signal transduction, but their clinical application is hurdled by the simultaneous 
induction of hyperthermia by TRPV1 blockade, potentially due to TRPV1's function in maintaining body temperature in central nervous system (Cui et al., 2006). It is interesting that long term or high dose treatment of TRPV1 agonists, such as capsaicin, cause receptor desensitization or kill the TRPV1 neurons, thus mimicking TRPV1 deficiency and leads to pain alleviation, suppression of TRPV1 mediated inflammation but without the conundrum of TRPV1 antagonists (Knotkova et al., 2008).

With the accumulation of evidence about the regulatory function of TRPV1 in the central nervous system for food intake and body temperature, as well as in peripheral organs for insulin and adipokine secretion, agonist-mediated TRPV1 desensitization begins to attract attention for its potential application in metabolic field. Capsaicin, as the classical TRPV1 agonist, has been shown in human and in rodents to increase satiety, reduce food intake, increase sympathetic nervous system activity (Reinbach et al., 2009; Janssens et al., 2014), and not surprisingly, enhance lipid metabolism (Kang et al., 2010) and activate thermogenesis (Baskaran et al., 2017). Mechanically, these reports consistently demonstrate that the metabolic effects of capsaicin no longer exist when TRPV1 is deficient, though capsaicin may also induce the Sirt1/PRDM16/PPAR $\gamma$ axis for its browning effects on white fat (Reinbach et al., 2009; Kang et al., 2010; Janssens et al., 2014; Baskaran et al., 2017). These findings signify the important potential of capsaicin and TRPV1 in the metabolic aspect. It has to be noted that other TPRV family members, i.e., TRPA1, TPRM8, and TRPV4, could be activated by different agonists/antagonists or sense different stimuli to exert various metabolic regulation, a particular intriguing example being TRPV4, which functions as a negative regulator of thermogenesis (Ye et al., 2012). Thus, aside from capsaicin, TCM would serve as a valuable source to screen more active compounds that target different TRPVs to open new therapeutic avenues in treating obesity and metabolic diseases.

\section{COMMON VS. RARE: BERBERINE AND GINSENOSIDES}

Traditional Chinese medicine contains medical extracts from a wide range of plants, animals and mineral products. In the long list, some are common everyday plants while some are rare and hard to cultivate. In drug development, after primary considerations like efficacy and safety are met, low development cost would be an advantage. A good example is Coptis chinensis and its active ingredient berberine, a common OTC drug with high popularity vs. ginseng, a TCM shrouded in mysterious atmosphere because of its effectiveness and rarity, though its development as a modern drug is hindered by its complex ingredients, ginsenosides.

\section{Berberine}

Coptis chinensis, a common and easy to cultivate medicinal herb, is one of the most widely used TCM with versatile effects since ancient China. The famous ancient Chinese proverb "a bitter medicine cures the disease," the bitter medicine referring to Coptis chinensis, exemplifies its long history of use and popularity in people. Coptis chinensis is routinely prescribed to treat bacteria induced diarrhea for its antibiotic properties. Today, it has been identified that the major active ingredient in Coptis chinensis is berberine. With the development and refinement of advanced synthesis technology, berberine is produced in large quantities and at low cost, thus becoming a standard collection in the medicine cabinet of most Chinese families. Besides diarrhea, in vitro and in vivo studies suggest that berberine is a potential drug in the treatment of type 2 diabetes mellitus, hyperlipidemia, and certain types of cancer (Liu et al., 2016). As early as 2004, Jiang's group has found that berberine binds to the $3^{\prime}$ UTR of LDLR mRNA, resulting in increased LDLR stability, enhanced hepatic LDL assimilation and reduced cholesterol level. In hypercholesterolemic patients and hyperlipidemic hamsters, berberine treatment significantly lowers total cholesterol, LDL and triglyceride levels with a mechanism of action distinct from Statins, a classic cholesterollowering drug targeting HMG-CoA reductase (Kong et al., 2004). Later, Ning's group showed the effectiveness of berberine in lipid and glycemic control in larger cohort of patients with Type 2 diabetes and hyperlipidemia based on comprehensive metabolomics (Zhang et al., 2008; Gu et al., 2010). Furthermore, berberine is shown to activate thermogenesis in adipocytes through the AMPK-PGC1 $\alpha$ axis, which leads to increased energy expenditure, reduced weight gain and improved cold tolerance in obese $\mathrm{db} / \mathrm{db}$ mice (Zhang et al., 2014).

Of note, berberine also exerts anti-aging function by mechanisms inhibiting mTOR/S6 pathway via AMPK activation, as well as reducing the endogenous ROS level and constitutive oxidative DNA damages through NRF2 (Halicka et al., 2012). In Drosophila melanogaster, berberine prolongs life span and stimulates locomotor activity potentially by blocking kynurenine formation from tryptophan, which is associated with aging (Navrotskaya et al., 2012). Since it has been shown that metabolic improvements are one of the major drives for longevity (Canaan et al., 2014; Ma et al., 2014), it would be interesting to assess how much of the extended longevity is contributed by berberine's promotion of metabolic health in the future and whether mammalian lifespan is also affected by berberine treatment.

In marked contrast to its effective in some clinical trials, the plasma level of berberine is found to be fairly low in patients (Hua et al., 2007), indicating that besides the classic pharmacological model, other factors might also in play in exerting berberine's beneficial effects. Promoted by sophisticated sequencing technologies, there has been a rapid growth in gut microbiota researches, spotlighting its critical involvement in the progression of multiple diseases including obesity and metabolic diseases. Due to its antimicrobial activity and poor solubility and absorption in the gut, berberine is put under the spotlight for its impacts on gut microbiota. As demonstrated by several independent groups, berberine improves high fat diet induced obesity and metabolic diseases through intestinal microbiota modulations (Han et al., 2011). Berberine treatment causes a structural change in gut microbial flora, largely reduces its diversity by enriching a few short-chain fatty acid (SCFA) producing bacteria including Blautia and Allobaculum, in turn elevates SCFA levels in the intestine and alleviates host 
inflammation (Zhang et al., 2012). Berberine also affects bile acid metabolism via its modulation on intestinal flora, by inhibiting cholic acid $7 \alpha$-dehydroxylation conversion into deoxycholic acid (Gu et al., 2015). Interestingly, in $\mathrm{ApoE}^{-/-}$atherosclerosis mice model, in addition to inducing a higher Akkermansia abundance in the gut flora, berberine administration is also found to preserve gut barrier integrity by increasing intestinal epithelial tight junction and colon mucus layer thickness in high fat diet-fed mice (Zhu et al., 2018). Last but not least, berberine could potentially promote metabolic health by playing a role in the microbiota-gutbrain axis. For example, berberine increases serum Glucagon-like Peptide-1 (GLP-1) and Neuropeptide Y level while decreases Orexin A level, which are all gut-brain peptides critical in satiety and energy homeostasis (Sun et al., 2016). Of note, GLP1 receptor is found to be elevated in the hypothalamus of berberine-treated mice, suggesting central nervous system might be another target for metabolic regulation by berberine (Sun et al., 2016).

\section{Ginsenosides}

Hidden in deep mountains, and difficult to find, ginseng is regarded by Chinese people as a rare and precious diet supplement to strengthen holistic health. Modern studies have shown that ginseng has anti-oxidant and anti-inflammation properties in cardiovascular and central nervous system, thus favoring healthy aging. The active ingredients in ginseng are ginsenosides, a class of steroid glycosides and triterpene saponins. There are more than 30 biologically active ginsenosides in ginseng, i.e., protopanaxadiols and protopanaxatriols, that all retain the beneficial effects of ginseng to some extent, thus it would be interesting to systemically assess the similarities and differences of these ginsenosides.

Numerous studies have demonstrated that ginsenosides are effective in preventing obesity, hyperlipidemia, hyperglycemia, and hepatic steatosis in DIO mice and rats, their action of targets including adipose tissue, liver, muscle and brain. In 3T3-L1 adipocytes, ginsenosides Rb1, Rg3, Rh1, Rf, and Re etc. suppress adipocyte differentiation by inhibiting the classical adipogenic transcription regulators PPAR $\gamma$ and C/EBPs (Lee et al., 2011; $\mathrm{Gu}$ et al., 2013; Siraj et al., 2015; Koh et al., 2017). In one independent research, $\mathrm{Rb} 1$ is shown to induce browning effects in 3T3-L1 adipocytes by increasing PPAR $\gamma$ activity, which is abrogated by PPAR $\gamma$ antagonist GW9692 treatment (Mu et al., 2015). Ginsenosides Rb1, Rg1, Rg5, and Re also target skeleton muscle for enhanced insulin sensitivity and cardiomyocytes for improved cardiac functions (Xie et al., 2006; Guan et al., 2017; Peng et al., 2017; Xiao et al., 2017b). In liver, Rg1, Rg3, Rg5, and $\mathrm{Rb} 2$ prevent hepatic steatosis with AMPK as their possible common target (Shen et al., 2013; Huang et al., 2017; Lee et al., 2017; Xiao et al., 2017a). There are also a few researches report that ginsenosides may target central nervous system in obese mice to improve leptin sensitivity in cortex and relieve central inflammation in hypothalamus (Wu et al., 2014).

Despite their consistent effectiveness in rodents and cellular models, meta-analysis of recent clinical trials assessing ginseng effects in cardiovascular diseases and metabolic diseases reveal that around $65 \%$ of the studies show significant improvements in ginseng treated groups, while the rest produce negative results
(Kim et al., 2015). For instance, one study has shown that ginseng itself and ginsenoside Re fail to improve $\beta$-cell function or insulin sensitivity in overweight and obese population with diabetes (Reeds et al., 2011). These inconsistencies might be due to the vast variants of ginseng species in North America and East Asia and the complexity of biologically active ginsenosides in different ginsengs. It would be worthwhile to invest more energy in isolating and characterizing the function of individual ginsenoside from various ginseng species, based on which more accurate clinical trials could be performed to assess their therapeutic potential in patients. Plus, developing efficient synthetic routes to obtain specific ginsenoside of interest would be of importance since extracting ginsenosides from ginseng might be expensive and low yield.

\section{Perspectives}

In this review, we have highlighted six compounds derived from TCM, artemisinin, curcumin, celastrol, capsaicin, berberine and ginsenoside, and evaluated various aspects of their property and their potentials in treating obesity and metabolic diseases (Figure 1). There are substantial clinical data on the safeness and side effects of artemisinin during its implication as a standard treatment for malaria. This makes it relatively easy to be transformed into a metabolic drug, although more data have to be obtained on its impact on metabolic parameters. On the other hand, clinical trials using curcumin have produced controversial results, which urge for more randomized, doubleblind, parallel controlled, multi-center clinical trials for fair judgment before it could be put into use. Celastrol, capsaicin and berberine are all promising novel therapeutics against obesity and metabolic diseases for their convincing effectiveness on metabolic improvement from in vitro and in vivo studies. Clinical data is required to access their efficacy and side effects on patients in the future. Ginseng, though famous for its holistic effects, has to be carefully analyzed to identify the detailed functions of individual ginsenosides in metabolism. Then, efforts are needed to find a cheaper way to synthesize the desired active ginsenosides. Besides the six compounds reviewed here, more TCM ingredients are waiting to be re-discovered and developed as novel drugs targeting obesity and metabolic diseases.

Along the history of mankind's everlasting pursuit for health, TCM plays an indispensable role with its unique yet empirical theory in diagnosing and treating diseases, which still holds significance in the modern point of view. Like the increased acceptance and popularity of Chinese ancient philosophy in western world, TCM theory is attracting more and more attention from the western medical establishment. For instance, TCM acknowledges the crosstalk among different organs and considers human body as a whole. Rather than targeting the affected parts alone, TCM strives to fight diseases in an integrated way. Its focus on complex interactions within biological systems coincides with the core idea of "Systems Biology," which is a powerful and fundamental tool for researches on subjects as complicated as human bodies. Secondly, similar to "Precision Medicine," TCM treatment is highly personalized. Ailments as simple as cold or headache are characterized based on different pathogenesis and 

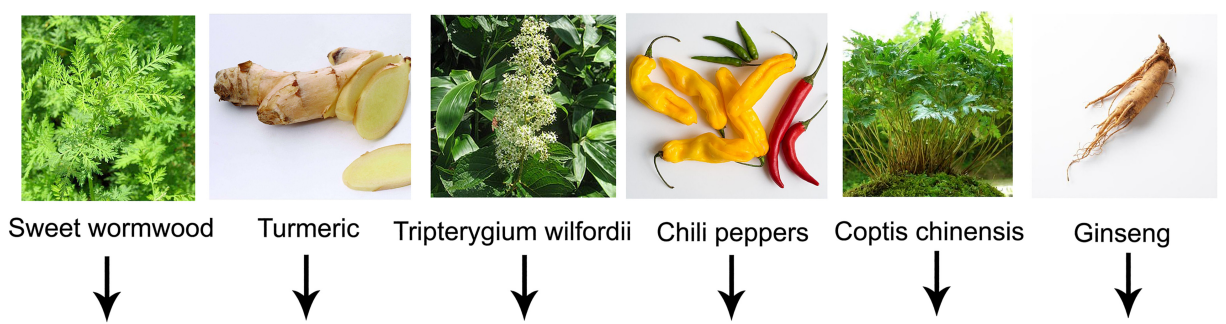

Sweet wormwood

Turmeric Tripterygium wilfordii
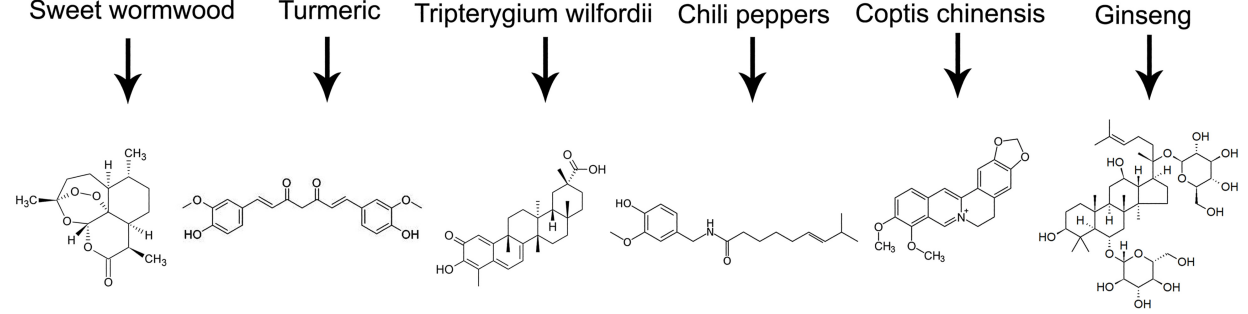

Artemisinin

Curcumin

Celastrol

Capsaicin

Berberine

Ginsenoside Rg1

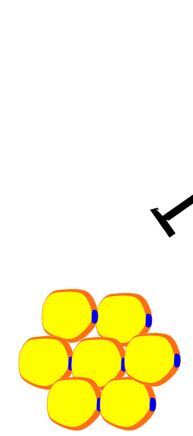

eWAT

Adipogenesis Inflammation $\downarrow$ Thermogenesis $\uparrow$

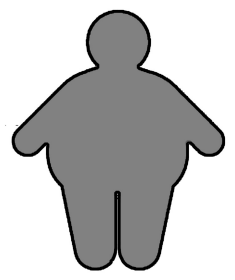

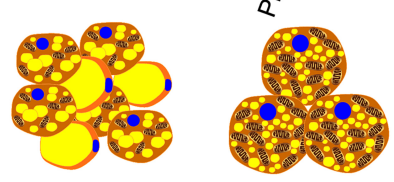

BAT
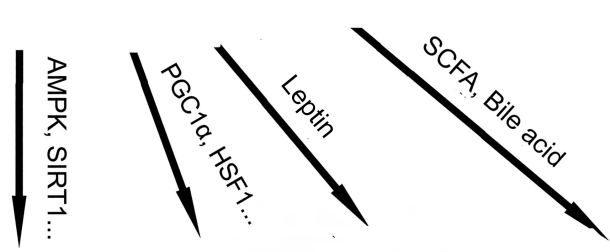

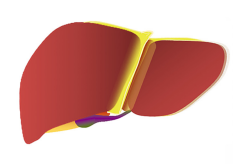

Liver

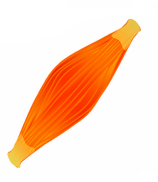

Muscle

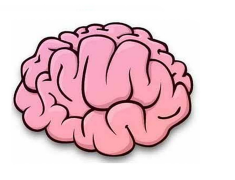

Brain

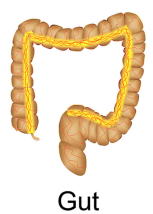

Gut

Inflammation $\downarrow \quad$ Mitochondrial $\uparrow \quad$ Leptin sensitivity $\uparrow$ Integrity $\uparrow$ Steatosis $\downarrow$ Insulin sensitivity $\uparrow \quad$ Food intake $\downarrow \quad$ GLP-1 $\uparrow$ Insulin sensitivity $\uparrow$

Body weights $\downarrow$ Hepatic steatosis $\downarrow$

Insulin sensitivity $\uparrow$

Hyperglycemia $\downarrow$

Hyperlipidemia $\downarrow$

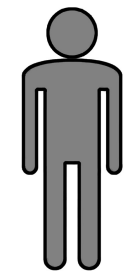

FIGURE 1 | Illustration summary of the original Traditional Chinese medicine (TCM) herbs, coumpound structures, metabolic effects and major mechanisms of artemisinin, curcumin, celastrol, capsaicin, berberine, and ginsenoside. eWAT, epidydimal white adipose tissue; iWAT, inguinal white adipose tissue; BAT, brown adipose tissue.

handled accordingly, rather than using a standard symptombased protocol as commonly practiced in western medicine. Other factors like patients' gender, sex, age, living condition, and lifestyle all play a role when design a prescription in TCM. It may seem chaotic at first impression, but it is clear now that a disease, especially obesity and metabolic diseases, is a highly interactive outcome of one's genetic and environment, thus the diagnostic strategy of TCM is not without solid foundation, although detailed mechanisms behind TCM theory have to be clearly addressed before it could be put into modern use. Thirdly, TCM formula is mostly Fufang (empirically combining multiple medicinal extracts to achieve best efficacy against a disease), which is similar to "Combination Therapy" in modern medicine.
Like in the case of celastrol and triptolide, TCM Fufang formula is a rich source to discover synergistic effects of numerous active ingredients to develop new combination therapy paradigm of better curative effects. Of course, similar to western drugs, TCM compounds have to be carefully reviewed and tested to decide the ideal treating strategy and eliminate side effects before they could be used as a therapeutic. To this end, a solid research and development protocol is indispensable to unravel the ancient secrets hidden in TCM to benefit patients in the modern world, at the same time prevents sporadic yet notorious incidence like Aristolochia and aristolochic acids in liver cancer (Ng et al., 2017).

Regarding the six candidate compounds discussed in this review, although in vitro mechanistic studies are extensive, it is 
of note that the effects of artemisinin and celastrol on metabolic parameters are evaluated in cellular and rodent models, which have limited extrapolation in human and require further clinical tests. Clinical studies on capcaisin and berberine produced positive data on metabolic fitness yet more studies are warranted, whereas the inconsistencies within the numerous clinical trials on curcumin and ginsenosides need reconciling. It is important to focus future studies on addressing these points to promote their translation into pharmaceuticals against obesity and metabolic diseases.

In summary, though great efforts are still needed to better understand its mechanisms and clinical relevance, TCM possesses great potential as a vast and readily available source for finding and developing new drugs against obesity and metabolic diseases. With the high prevalence of obesity and metabolic diseases in population and the resulting high cost for diseases care, it would be wise to devote

\section{REFERENCES}

Anckar, J., and Sistonen, L. (2011). Regulation of HSF1 function in the heat stress response: implications in aging and disease. Annu. Rev. Biochem. 80, 1089-1115. doi: 10.1146/annurev-biochem-060809095203

Baker, M. (2017). Deceptive curcumin offers cautionary tale for chemists. Nature 541, 144-145. doi: 10.1038/541144a

Baskaran, P., Krishnan, V., Fettel, K., Gao, P., Zhu, Z., Ren, J., et al. (2017). TRPV1 activation counters diet-induced obesity through sirtuin-1 activation and PRDM-16 deacetylation in brown adipose tissue. Int. J. Obes. 41, 739-749. doi: $10.1038 /$ ijo.2017.16

Bays, H., and Dujovne, C. A. (2006). Adiposopathy is a more rational treatment target for metabolic disease than obesity alone. Curr. Atheroscler. Rep. 8, $144-156$.

Boss, O., and Farmer, S. R. (2012). Recruitment of brown adipose tissue as a therapy for obesity-associated diseases. Front. Endocrinol. 3:14. doi: 10.3389/ fendo.2012.00014

Canaan, A., DeFuria, J., Perelman, E., Schultz, V., Seay, M., Tuck, D., et al. (2014). Extended lifespan and reduced adiposity in mice lacking the FAT10 gene. Proc. Natl. Acad. Sci. U.S.A. 111, 5313-5318. doi: 10.1073/pnas.1323426111

Cascão, R., Fonseca, J. E., and Moita, L. F. (2017). Celastrol: a spectrum of treatment opportunities in chronic diseases. Front. Med. 4:69. doi: 10.3389/ fmed.2017.00069

Caterina, M. J., Schumacher, M. A., Tominaga, M., Rosen, T. A., Levine, J. D., and Julius, D. (1997). The capsaicin receptor: a heat-activated ion channel in the pain pathway. Nature 389, 816-824. doi: 10.1038/39807

Corson, T. W., and Crews, C. M. (2007). Molecular understanding and modern application of traditional medicines: triumphs and trials. Cell 130, 769-774. doi: 10.1016/j.cell.2007.08.021

Cui, M., Honore, P., Zhong, C., Gauvin, D., Mikusa, J., Hernandez, G., et al. (2006). TRPV1 receptors in the CNS play a key role in broad-spectrum analgesia of TRPV1 antagonists. J. Neurosci. 26, 9385-9393. doi: 10.1523/JNEUROSCI. 1246-06.2006

Cypess, A. M., and Kahn, C. R. (2010). Brown fat as a therapy for obesity and diabetes. Curr. Opin. Endocrinol. Diabetes Obes. 17, 143-149. doi: 10.1097/ MED.0b013e328337a81f

Ejaz, A., Wu, D., Kwan, P., and Meydani, M. (2009). Curcumin inhibits adipogenesis in 3T3-L1 adipocytes and angiogenesis and obesity in C57/BL mice. J. Nutr. 139, 919-925. doi: 10.3945/jn.108.100966

Ganjali, S., Sahebkar, A., Mahdipour, E., Jamialahmadi, K., Torabi, S., Akhlaghi, S., et al. (2014). Investigation of the effects of curcumin on serum cytokines in obese individuals: a randomized controlled trial. Scientific World J. 2014:898361. doi: 10.1155/2014/898361 more resources in researching of TCM for new therapeutic inspirations.

\section{AUTHOR CONTRIBUTIONS}

LX and XM conceived the review and LX, WZ, DW, and XM wrote the manuscript.

\section{FUNDING}

This project was supported by funding from National Natural Science Foundation of China (31770840), The Program for Professor of Special Appointment (Eastern Scholar) at Shanghai Institutions of Higher Learning, Shanghai Pujiang Program (17PJ1402700 and 17PJ1402600) and China Postdoctoral Science Foundation (2017M611499).

Gavva, N. R. (2008). Body-temperature maintenance as the predominant function of the vanilloid receptor TRPV1. Trends Pharmacol. Sci. 29, 550-557. doi: 10.1016/j.tips.2008.08.003

Gomez-Pastor, R., Burchfiel, E. T., and Thiele, D. J. (2018). Regulation of heat shock transcription factors and their roles in physiology and disease. Nat. Rev. Mol. Cell Biol. 19, 4-19. doi: 10.1038/nrm.2017.73

Gu, S., Cao, B., Sun, R., Tang, Y., Paletta, J. L., Wu, X., et al. (2015). A metabolomic and pharmacokinetic study on the mechanism underlying the lipid-lowering effect of orally administered berberine. Mol. Biosyst. 11, 463-474. doi: 10.1039/ c4mb00500g

Gu, W., Kim, K. A., and Kim, D. H. (2013). Ginsenoside Rh1 ameliorates high fat diet-induced obesity in mice by inhibiting adipocyte differentiation. Biol. Pharm. Bull. 36, 102-107. doi: 10.1248/bpb.b1200558

Gu, Y., Zhang, Y., Shi, X., Li, X., Hong, J., Chen, J., et al. (2010). Effect of traditional Chinese medicine berberine on type 2 diabetes based on comprehensive metabonomics. Talanta 81, 766-772. doi: 10.1016/j.talanta.2010. 01.015

Guan, S., Xu, W., Han, F., Gu, W., Song, L., Ye, W., et al. (2017). Ginsenoside Rg1 attenuates cigarette smoke-induced pulmonary epithelial-mesenchymal transition via inhibition of the TGF- $\beta /$ Smad pathway. Biomed Res. Int. 2017:7171404. doi: 10.1155/2017/7171404

Gunthorpe, M. J., and Szallasi, A. (2008). Peripheral TRPV1 receptors as targets for drug development: new molecules and mechanisms. Curr. Pharm. Des. 14, 32-41. doi: 10.2174/138161208783330754

Gupta, A., Singh, V. K., Kumar, D., Yadav, P., Kumar, S., Beg, M., et al. (2017). Curcumin-3,4-Dichloro Phenyl Pyrazole (CDPP) overcomes curcumin's low bioavailability, inhibits adipogenesis and ameliorates dyslipidemia by activating reverse cholesterol transport. Metabolism 73, 109-124.

Halicka, H. D., Zhao, H., Li, J., Lee, Y. S., Hsieh, T. C., Wu, J. M., et al. (2012). Potential anti-aging agents suppress the level of constitutive mTOR- and DNA damage- signaling. Aging 4, 952-965. doi: 10.18632/aging. 100521

Han, J., Lin, H., and Huang, W. (2011). Modulating gut microbiota as an anti-diabetic mechanism of berberine. Med. Sci. Monit. 17, RA164-RA167. doi: 10.12659/MSM.881842

Haslam, D. W., and James, W. P. (2005). Obesity. Lancet 366, 1197-1209. doi: 10.1016/S0140-6736(05)67483-1

Heger, M. (2017). Drug screening: Don't discount all curcumin trial data. Nature 543:40. doi: $10.1038 / 543040 \mathrm{c}$

Hu, M., Luo, Q., Alitongbieke, G., Chong, S., Xu, C., Xie, L., et al. (2017). Celastrolinduced Nur77 interaction with TRAF2 alleviates inflammation by promoting mitochondrial ubiquitination and autophagy. Mol. Cell 66, 141.e6-153.e6. doi: 10.1016/j.molcel.2017.03.008 
Hua, W., Ding, L., Chen, Y., Gong, B., He, J., and Xu, G. (2007). Determination of berberine in human plasma by liquid chromatography-electrospray ionizationmass spectrometry. J. Pharm. Biomed. Anal. 44, 931-937. doi: 10.1016/j.jpba. 2007.03.022

Huang, Q., Wang, T., Yang, L., and Wang, H. Y. (2017). Ginsenoside Rb2 alleviates hepatic lipid accumulation by restoring autophagy via induction of Sirt1 and activation of AMPK. Int. J. Mol. Sci. 18, E1063. doi: 10.3390/ijms18051063

Jang, B. C. (2016). Artesunate inhibits adipogeneis in 3T3-L1 preadipocytes by reducing the expression and/or phosphorylation levels of C/EBP- $\alpha$, PPAR- $\gamma$, FAS, perilipin A, and STAT-3. Biochem. Biophys. Res. Commun. 474, 220-225. doi: 10.1016/j.bbrc.2016.04.109

Janssens, P. L., Hursel, R., and Westerterp-Plantenga, M. S. (2014). Capsaicin increases sensation of fullness in energy balance, and decreases desire to eat after dinner in negative energy balance. Appetite 77, 44-49. doi: 10.1016/j.appet. 2014.02.018

Jiang, Q. W., Cheng, K. J., Mei, X. L., Qiu, J. G., Zhang, W. J., Xue, Y. Q., et al. (2015). Synergistic anticancer effects of triptolide and celastrol, two main compounds from thunder god vine. Oncotarget 6, 32790-32804. doi: 10.18632/ oncotarget.5411

Kang, J. H., Goto, T., Han, I. S., Kawada, T., Kim, Y. M., and Yu, R. (2010). Dietary capsaicin reduces obesity-induced insulin resistance and hepatic steatosis in obese mice fed a high-fat diet. Obesity 18, 780-787. doi: 10.1038/oby.2009.301

Kim, C. Y., Le, T. T., Chen, C., Cheng, J. X., and Kim, K. H. (2011), Curcumin inhibits adipocyte differentiation through modulation of mitotic clonal expansion. J. Nutr. Biochem. 22, 910-920. doi: 10.1016/j.jnutbio.2010. 08.003

Kim, K. E., Ko, K. H., Heo, R. W., Yi, C. O., Shin, H. J., Kim, J. Y., et al. (2016). Artemisia annua leaf extract attenuates hepatic steatosis and inflammation in high-fat diet-fed mice. J. Med. Food 19, 290-299. doi: 10.1089/jmf.2015.3527

Kim, Y. S., Woo, Y. Y., Han, C. K., and Chang, I. M. (2015). Safety analysis of panax ginseng in randomized clinical trials: a systematic review. Medicines 2, 106-126. doi: 10.3390/medicines2020106

Knotkova, H., Pappagallo, M., and Szallasi, A. (2008). Capsaicin (TRPV1 Agonist) therapy for pain relief: farewell or revival? Clin. J. Pain 24, 142-154. doi: 10.1097/AJP.0b013e318158ed9e

Koh, E., Kim, K. J., Choi, J., Jeon, H. J., Seo, M. J., and Lee, B. Y. (2017). Ginsenoside Rg1 suppresses early stage of adipocyte development via activation of C/EBP homologous protein-10 in 3T3-L1 and attenuates fat accumulation in high fat diet-induced obese zebrafish. J. Ginseng Res. 41, 23-30. doi: 10.1016/j.jgr.2015. 12.005

Kong, W., Wei, J., Abidi, P., Lin, M., Inaba, S., Li, C., et al. (2004). Berberine is a novel cholesterol-lowering drug working through a unique mechanism distinct from statins. Nat. Med. 10, 1344-1351. doi: 10.1038/nm1135

Lee, J., Kim, M. H., Lee, J. H., Jung, E., Yoo, E. S., and Park, D. (2012). Artemisinic acid is a regulator of adipocyte differentiation and C/EBP $\delta$ expression. J. Cell. Biochem. 113, 2488-2499. doi: 10.1002/jcb.24124

Lee, J. B., Yoon, S. J., Lee, S. H., Lee, M. S., Jung, H., Kim, T. D., et al. (2017). Ginsenoside Rg3 ameliorated HFD-induced hepatic steatosis through downregulation of STAT5-PPAR $\gamma$. J. Endocrinol. 235, 223-235. doi: 10.1530/ JOE-17-0233

Lee, O. H., Lee, H. H., Kim, J. H., and Lee, B. Y. (2011). Effect of ginsenosides Rg3 and Re on glucose transport in mature 3T3-L1 adipocytes. Phytother. Res. 25, 768-773. doi: $10.1002 /$ ptr.3322

Li, X. J., Jiang, Z. Z., and Zhang, L. Y. (2014). Triptolide: progress on research in pharmacodynamics and toxicology. J. Ethnopharmacol. 155, 67-79. doi: 10.1016/j.jep.2014.06.006

Liu, C., Zhao, Y., and Wang, Y. (2006). Artemisinin: current state and perspectives for biotechnological production of an antimalarial drug. Appl. Microbiol. Biotechnol. 72, 11-20. doi: 10.1007/s00253-006-0452-0

Liu, C. S., Zheng, Y. R., Zhang, Y. F., and Long, X. Y. (2016). Research progress on berberine with a special focus on its oral bioavailability. Fitoterapia 109, 274-282. doi: 10.1016/j.fitote.2016.02.001

Liu, J., Lee, J., Salazar Hernandez, M. A., Mazitschek, R., and Ozcan, U. (2015). Treatment of obesity with celastrol. Cell 161, 999-1011. doi: 10.1016/j.cell.2015. 05.011

Lo-Coco, F., Avvisati, G., Vignetti, M., Thiede, C., Orlando, S. M., Iacobelli, S., et al. (2013). Retinoic acid and arsenic trioxide for acute promyelocytic leukemia. N. Engl. J. Med. 369, 111-121. doi: 10.1056/NEJMoa1300874
Lone, J., Choi, J. H., Kim, S. W., and Yun, J. W. (2016). Curcumin induces brown fat-like phenotype in 3T3-L1 and primary white adipocytes. J. Nutr. Biochem. 27, 193-202. doi: 10.1016/j.jnutbio.2015.09.006

Lopez, L. M., Grimes, D. A., and Schulz, K. F. (2005). Nonhormonal drugs for contraception in men: a systematic review. Obstet. Gynecol. Surv. 60, 746-752. doi: 10.1097/01.ogx.0000182905.71077.13

Lu, P., Zhang, F. C., Qian, S. W., Li, X., Cui, Z. M., Dang, Y. J., et al. (2016). Artemisinin derivatives prevent obesity by inducing browning of WAT and enhancing BAT function. Cell Res. 26, 1169-1172. doi: 10.1038/cr.2016.108

Luo, D., Guo, Y., Cheng, Y., Zhao, J., Wang, Y., and Rong, J. (2017). Natural product celastrol suppressed macrophage M1 polarization against inflammation in diet-induced obese mice via regulating $\mathrm{Nrf} / \mathrm{HO}-1$, MAP kinase and NF-кB pathways. Aging 9, 2069-2082. doi: 10.18632/aging.101302

Ma, X., Xu, L., Alberobello, A. T., Gavrilova, O., Bagattin, A., Skarulis, M., et al. (2015). Celastrol protects against obesity and metabolic dysfunction through activation of a HSF1-PGC1 $\alpha$ transcriptional axis. Cell Metab. 22, 695-708. doi: 10.1016/j.cmet.2015.08.005

Ma, X., Xu, L., Gavrilova, O., and Mueller, E. (2014). Role of forkhead box protein A3 in age-associated metabolic decline. Proc. Natl. Acad. Sci. U.S.A. 111 14289-14294. doi: 10.1073/pnas.1407640111

Mohammadi, A., Sahebkar, A., Iranshahi, M., Amini, M., Khojasteh, R., GhayourMobarhan, M., et al. (2013). Effects of supplementation with curcuminoids on dyslipidemia in obese patients: a randomized crossover trial. Phytother. Res. 27, 374-379. doi: 10.1002/ptr.4715

Mu, Q., Fang, X., Li, X., Zhao, D., Mo, F., Jiang, G., et al. (2015). Ginsenoside $\mathrm{Rb} 1$ promotes browning through regulation of PPAR $\gamma$ in 3T3-L1 adipocytes. Biochem. Biophys. Res. Commun. 466, 530-535. doi: 10.1016/j.bbrc.2015.09.064

Navrotskaya, V. V., Oxenkrug, G., Vorobyova, L. I., and Summergrad, P. (2012). Berberine prolongs life span and stimulates locomotor activity of Drosophila melanogaster. Am. J. Plant Sci. 3, 1037-1040. doi: 10.4236/ajps.2012.327123

Nelson, K. M., Dahlin, J. L., Bisson, J., Graham, J., Pauli, G. F., and Walters, M. A. (2017). The essential medicinal chemistry of curcumin. J. Med. Chem 60, 1620-1637. doi: 10.1021/acs.jmedchem.6b00975

Ng, A. W. T., Poon, S. L., Huang, M. N., Lim, J. Q., Boot, A., Yu, W., et al. (2017). Aristolochic acids and their derivatives are widely implicated in liver cancers in Taiwan and throughout Asia. Sci. Transl. Med. 9:eaan6446. doi: 10.1126/scitranslmed.an6446

Pan, Y., Wang, Y., Zhao, Y., Peng, K., Li, W., Wang, Y., et al. (2014). Inhibition of JNK phosphorylation by a novel curcumin analog prevents high glucoseinduced inflammation and apoptosis in cardiomyocytes and the development of diabetic cardiomyopathy. Diabetes Metab. Res. Rev. 63, 3497-3511. doi: $10.2337 / \mathrm{db} 13-1577$

Peng, Y., Zhang, R., Kong, L., Shen, Y., Xu, D., Zheng, F., et al. (2017). Ginsenoside Rg3 inhibits the senescence of prostate stromal cells through down-regulation of interleukin 8 expression. Oncotarget 8, 64779-64792. doi: $10.18632 /$ oncotarget.17616

Priyanka, A., Shyni, G. L., Anupama, N., Raj, P. S., Anusree, S. S., and Raghu, K. G. (2017). Development of insulin resistance through sprouting of inflammatory markers during hypoxia in 3T3-L1 adipocytes and amelioration with curcumin. Eur. J. Pharmacol. 812, 73-81. doi: 10.1016/j.ejphar.2017.07.005

Qiu, J. (2007). Traditional medicine: a culture in the balance. Nature 448, 126-128. doi: $10.1038 / 448126 a$

Rahimi, H. R., Nedaeinia, R., Sepehri Shamloo, A., Nikdoust, S., and Kazemi Oskuee, R. (2016). Novel delivery system for natural products: nano-curcumin formulations. Avicenna J. Phytomed. 6, 383-398.

Reeds, D. N., Patterson, B. W., Okunade, A., Holloszy, J. O., Polonsky, K. S and Klein, S. (2011). Ginseng and ginsenoside Re do not improve $\beta$-cell function or insulin sensitivity in overweight and obese subjects with impaired glucose tolerance or diabetes. Diabetes Care 34, 1071-1076. doi: 10.2337/ dc10-2299

Reinbach, H. C., Smeets, A., Martinussen, T., Møller, P., and Westerterp-Plantenga, M. S. (2009). Effects of capsaicin, green tea and $\mathrm{CH}-19$ sweet pepper on appetite and energy intake in humans in negative and positive energy balance. Clin. Nutr. 28, 260-265. doi: 10.1016/j.clnu.2009.01.010

Sakuma, S., Sumida, M., Endoh, Y., Kurita, A., Yamaguchi, A., Watanabe, T., et al. (2017). Curcumin inhibits adipogenesis induced by benzyl butyl phthalate in 3T3-L1 cells. Toxicol. Appl. Pharmacol. 329, 158-164. doi: 10.1016/j.taap.2017. 05.036 
Saltiel, A. R., and Olefsky, J. M. (2017). Inflammatory mechanisms linking obesity and metabolic disease. J. Clin. Invest. 127, 1-4. doi: 10.1172/JCI92035

Shao, W., Yu, Z., Chiang, Y., Yang, Y., Chai, T., Foltz, W., et al. (2012). Curcumin prevents high fat diet induced insulin resistance and obesity via attenuating lipogenesis in liver and inflammatory pathway in adipocytes. PLoS One 7:e28784. doi: 10.1371/journal.pone.0028784

Shen, L., Xiong, Y., Wang, D. Q., Howles, P., Basford, J. E., Wang, J., et al. (2013). Ginsenoside Rb1 reduces fatty liver by activating AMP-activated protein kinase in obese rats. J. Lipid Res. 54, 1430-1438. doi: 10.1194/jlr.M035907

Siraj, F. M., Natarajan, S., Huq, M. A., Kim, Y. J., and Yang, D. C. (2015). Structural investigation of ginsenoside Rf with $\operatorname{PPAR} \gamma$ major transcriptional factor of adipogenesis and its impact on adipocyte. J. Ginseng Res. 39, 141-147. doi: 10.1016/j.jgr.2014.10.002

Sun, H., Wang, N., Cang, Z., Zhu, C., Zhao, L., Nie, X., et al. (2016). Modulation of microbiota-gut-brain axis by berberine resulting in improved metabolic status in high-fat diet-fed rats. Obes. Facts 9, 365-378. doi: 10.1159/0004 49507

Wang, S., Wang, X., Ye, Z., Xu, C., Zhang, M., Ruan, B., et al. (2015). Curcumin promotes browning of white adipose tissue in a norepinephrine-dependent way. Biochem. Biophys. Res. Commun. 466, 247-253. doi: 10.1016/j.bbrc.2015.09.018

Weisberg, S. P., Leibel, R., and Tortoriello, D. V. (2008). Dietary curcumin significantly improves obesity-associated inflammation and diabetes in mouse models of diabesity. Endocrinology 149, 3549-3558. doi: 10.1210/en.2008-0262

Westerheide, S. D., Bosman, J. D., Mbadugha, B. N., Kawahara, T. L., Matsumoto, G., Kim, S., et al. (2004). Celastrols as inducers of the heat shock response and cytoprotection. J. Biol. Chem. 279, 56053-56060. doi: 10.1074/jbc. M409267200

Wu, Y., Yu, Y., Szabo, A., Han, M., and Huang, X. F. (2014). Central inflammation and leptin resistance are attenuated by ginsenoside $\mathrm{Rb} 1$ treatment in obese mice fed a high-fat diet. PLoS One 9:e92618. doi: 10.1371/journal.pone.0092618

Xiao, N., Lou, M. D., Lu, Y. T., Yang, L. L., Liu, Q., Liu, B., et al. (2017a). Ginsenoside $\operatorname{Rg} 5$ attenuates hepatic glucagon response via suppression of succinate-associated HIF- $1 \alpha$ induction in HFD-fed mice. Diabetologia 60, 1084-1093. doi: 10.1007/s00125-017-4238-y

Xiao, N., Yang, L. L., Yang, Y. L., Liu, L. W., Li, J., Liu, B., et al. (2017b). Ginsenoside Rg5 inhibits succinate-associated lipolysis in adipose tissue and prevents muscle insulin resistance. Front. Pharmacol. 8:43. doi: 10.3389/fphar.2017.00043
Xie, J. T., Shao, Z. H., Vanden Hoek, T. L., Chang, W. T., Li, J., Mehendale, S., et al. (2006). Antioxidant effects of ginsenoside Re in cardiomyocytes. Eur. J. Pharmacol. 532, 201-207. doi: 10.1016/j.ejphar.2006.01.001

Xu, L., Ma, X., Verma, N. K., Wang, D., Gavrilova, O., Proia, R. L., et al. (2018). Ablation of PPAR $\gamma$ in subcutaneous fat exacerbates age-associated obesity and metabolic decline. Aging Cell 17, e12721. doi: 10.1111/acel.12721

Ye, L., Kleiner, S., Wu, J., Sah, R., Gupta, R. K., Banks, A. S., et al. (2012). TRPV4 is a regulator of adipose oxidative metabolism, inflammation, and energy homeostasis. Cell 151, 96-110. doi: 10.1016/j.cell.2012.08.034

Zhang, X., Zhao, Y., Zhang, M., Pang, X., Xu, J., Kang, C., et al. (2012). Structural changes of gut microbiota during berberine-mediated prevention of obesity and insulin resistance in high-fat diet-fed rats. PLoS One 7:e42529. doi: 10.1371/ journal.pone.0042529

Zhang, Y., Geng, C., Liu, X., Li, M., Gao, M., Liu, X., et al. (2016). Celastrol ameliorates liver metabolic damage caused by a high-fat diet through Sirt1. Mol. Metab. 6, 138-147. doi: 10.1016/j.molmet.2016.11.002

Zhang, Y., Li, X., Zou, D., Liu, W., Yang, J., Zhu, N., et al. (2008). Treatment of type 2 diabetes and dyslipidemia with the natural plant alkaloid berberine. J. Clin. Endocrinol. Metab. 93, 2559-2565. doi: 10.1210/jc.2007-2404

Zhang, Z., Zhang, H., Li, B., Meng, X., Wang, J., Zhang, Y., et al. (2014). Berberine activates thermogenesis in white and brown adipose tissue. Nat. Commun 5:5493. doi: $10.1038 /$ ncomms6493

Zhu, L., Zhang, D., Zhu, H., Zhu, J., Weng, S., Dong, L., et al. (2018). Berberine treatment increases Akkermansia in the gut and improves high-fat diet-induced atherosclerosis in Apoe ${ }^{-} /^{-}$mice. Atherosclerosis 268, 117-126. doi: 10.1016/j. atherosclerosis.2017.11.023

Conflict of Interest Statement: The authors declare that the research was conducted in the absence of any commercial or financial relationships that could be construed as a potential conflict of interest.

Copyright (c) $2018 \mathrm{Xu}$, Zhao, Wang and Ma. This is an open-access article distributed under the terms of the Creative Commons Attribution License (CC BY). The use, distribution or reproduction in other forums is permitted, provided the original author(s) and the copyright owner(s) are credited and that the original publication in this journal is cited, in accordance with accepted academic practice. No use distribution or reproduction is permitted which does not comply with these terms. 